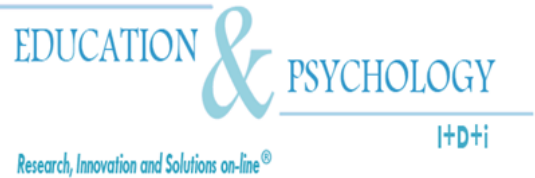

\title{
Intervención familiar en niños con trastornos del lenguaje: Una revisión.
}

\author{
Ester Ato Lozano ${ }^{1}, \mathrm{M}^{\mathrm{a}}$ Dolores Galián Conesa ${ }^{2}$, \\ Francisco Cabello Luque ${ }^{1}$ \\ ${ }^{1}$ Facultad de Psicología, Universidad de Murcia \\ ${ }^{2}$ Facultad de Psicología, Universidad de Elche
}

\section{España}

Correspondencia: Ester Ato Lozano. Facultad de Psicología. Campus de Espinardo. Murcia. España. E-mail: esterato@um.es

(C) Education \& Psychology $\mathrm{I}+\mathrm{D}+\mathrm{i}$ and Editorial EOS (Spain) 


\section{Resumen}

La perspectiva sociointeraccionista considera a la familia como el escenario fundamental donde el niño puede aprender el lenguaje, y por tanto un elemento clave en el proceso de intervención sobre las alteraciones en el desarrollo del mismo. Este trabajo tiene como objetivo la revisión teórica de los principales estudios sobre intervención familiar en niños con trastornos del lenguaje desde esta perspectiva, comparando la efectividad de distintos tipos de programas centrados en la familia y la terapia logopédica habitual en el trastorno específico del lenguaje (TEL) y en los déficits de lenguaje asociados a patologías como el autismo, la discapacidad auditiva y el retraso cognitivo. Los resultados muestran que la intervención centrada en la familia produce mejoras en el rendimiento lingüístico cuanto menos similares a los de la terapia habitual administrada por un profesional. Además, los beneficios de esta intervención no son sólo relativos al área del lenguaje, sino también al área psicológica y emocional de todos los miembros de la familia.

Palabras Clave: familia, lenguaje, intervención en lenguaje, modelo sociointeraccionista, trastorno específico del lenguaje, autismo, sordera, retraso mental, niños.

Recibido: 04/05/09 Aceptación inicial: 08/05/09 Aceptación final: 16/09/09 


\title{
Family intervention in children with language disabilities: a review
}

\begin{abstract}
From a social-interaccionistic perspective, family can be view as the main setting where children learn and develop language, and thus it is considered a key element in the intervention on language development impairment. This paper is aimed at providing, from this perspective, a theoretical review of the main studies on family intervention in children with language impairments. A comparison is established between different types of family-centered intervention programs and the usual speech-therapy treatments for children with specific language impairment (SLI) other pathologies such as autism, hearing impairments and cognitive delay. Results indicate that family-centered intervention produced improvements in the language of children that are closely similar to those found in therapeutic programs administered by a professional therapist. Furthermore, family-centered interventions did not only improve the language of children, but also showed to produce psychological and emotional benefits for all family members.
\end{abstract}

Keywords: family, language, language intervention, social-interaccionistic model, specific language impairment, autism, hearing impairment, mental delay, children.

Received: 05/04/09 Initial Acceptance: 05/08/09 Final Acceptance: 09/16/09 


\section{Introducción}

En la actualidad existen multitud de estudios que corroboran un aumento de la prevalencia de dificultades asociadas al lenguaje, permanentes o transitorias, en niños diagnosticados con "necesidades educativas especiales" (Acosta, 2004). Este tipo de trastornos se han asociado a su vez a una mayor frecuencia de conductas desadaptativas en las áreas social, emocional y académica, entre otras (Goldstein y Kaczmarek, 1992; van Daal, Verhoeven y van Balkom, 2007; Warren y Kaiser, 1986). Desde esta perspectiva, se plantea un creciente interés por la descripción y análisis de aquellas variables que intervienen en el proceso de adquisición del lenguaje, normal y patológico. Una de las variables que se ha mostrado más potente a la hora de explicar la génesis y desarrollo del lenguaje y sus alteraciones es la interacción social (Bruner, 1983; Grácia, 2002; Nelson, 1977).

Así, los cuidadores no solo determinan en gran parte la forma en que el niño adquiere el lenguaje, sino que tienen un peso relevante en la posible evolución de las dificultades asociadas al mismo. No es de extrañar, por tanto, que en el estudio de los trastornos del lenguaje se considere a la familia un factor a tener en cuenta, especialmente en la fase de tratamiento o intervención. Este papel puede variar según el planteamiento de la intervención, pudiendo ser meros informantes o apoyos externos al logopeda, que dirige la intervención; o ser agentes activos altamente implicados en todas las fases del proceso. Desde esta última perspectiva, se parte de la idea de que el niño responde mejor ante intervenciones centradas en el contexto familiar, alejadas de planteamientos altamente estructurados con adultos desconocidos (Schertz y Odom, 2007). Históricamente, ha variado el tipo de programa aplicado desde el ámbito familiar, aumentado con los años la búsqueda de mayor naturalidad en las estrategias y contextos asociados a la intervención, además de un mayor rigor metodológico en su aplicación (Girolametto y Weitzman, 2008).

Multitud de estudios han intentado probar la eficacia de los programas centrados en la familia. Tanto en los trastornos específicos del lenguaje, como en los trastornos del lenguaje asociados a otras patologías, parece probada la mejora en el rendimiento lingüístico de los niños tras la intervención familiar, en las áreas fonológica, semántica, morfosintáctica y pragmática (Bowen y Cupples, 1999; Coulter y Gallhager, 2001; Hemmeter y Kaiser, 1994; Moller, Probst y Hess, 2008). Los estudios que comparan este tipo de intervención con la tradicional, han encontrado, cuanto menos, niveles similares de ganancia lingüística en ambos 
grupos (Baxendale y Hesketh, 2003; Gibbard, Coglan y McDonald, 2004). No obstante, en algunos estudios se detectan errores metodológicos que dificultan el establecimiento de causalidad entre la acción de los padres y los progresos obtenidos por los niños. La investigación empírica parece probar, en cualquier caso, la mejoría tanto en aspectos propiamente lingüísticos, como psicológicos y emocionales, de la participación de la familia en el proceso de intervención (Lederer, 2001; Niccols y Mohamed, 2000).

Este trabajo realiza una revisión de las investigaciones que analizan el papel de la familia en la intervención de los trastornos del lenguaje, abordando tanto cuestiones de tipo conceptual como empíricas.

\section{El papel de la familia en la intervención de los trastornos del lenguaje: Tipos de inter- vención.}

La intervención de los trastornos del lenguaje se ha abordado desde distintas perspectivas. Tradicionalmente, se ha considerado al logopeda o especialista en lenguaje como la persona encargada de intervenir directamente sobre los problemas lingüísticos del niño. No obstante, dada la tendencia que se observa a la búsqueda de una detección e intervención temprana de los trastornos del lenguaje, los padres han empezado a cobrar un mayor protagonismo en los programas de intervención. Este mayor protagonismo se traduce en una colaboración estrecha con el terapeuta en el diseño y actuación del plan de intervención, o incluso, en una asunción por parte de los padres del rol de terapeutas.

Teniendo en cuenta este protagonismo, podemos establecer una clasificación de las intervenciones sobre las alteraciones del lenguaje en niños según el papel de los padres: intervenciones en que el terapeuta asume el mayor protagonismo, intervenciones en las que la familia es un agente colaborador, e intervenciones en las que la familia es el agente fundamental de intervención.

A continuación vamos a describir más detalladamente el papel asumido por los padres en cada uno de estos tipos de intervención 
La aproximación centrada en el terapeuta o logopeda.

Desde este modelo de intervención, el terapeuta es el máximo responsable de la implementación del plan de intervención. Es la persona que evalúa y diagnostica al niño, la que planifica e implementa los objetivos, la que plantea las estrategias a seguir, y la que directamente actúa con el niño.

En esta forma de trabajo, los padres son meros testigos del proceso de intervención, y, en el mejor de los casos, ayudan a los terapeutas a evaluar el estado inicial del niño, o constatan los progresos observados tras la intervención en el contexto familiar. No obstante, los padres no se implican en ninguna de las fases del proceso de intervención, ni su opinión es tenida en cuenta para el diseño de los objetivos, estrategias y plan de actuación. La terapia en estos casos se suele realizar en el ámbito de la clínica privada, o en el contexto escolar, y se administra de forma individualizada, o en pequeños grupos. Una de las críticas que se ha realizado a este tipo de intervención es la ausencia de naturalidad desde la que se plantea, por lo que su generalización a otros contextos suele ser dificultosa para el niño (Vilaseca y Del Río, 1997).

Existen numerosos estudios que han probado los efectos de la intervención logopédica en niños con alteraciones del lenguaje (Law y Conti-Ramsden, 2000; Tallal y Rice, 1998). En concreto, un estudio metaanalítico reciente ha analizado la eficacia de la evaluación logopédica en niños con trastornos del lenguaje (Law, Garrett y Nye, 2004), y ha encontrado que los resultados de esta intervención son más óptimos cuando los niños presentan problemas fonológicos o del lenguaje expresivo, mientras que la efectividad no es tan clara cuando los problemas son sintácticos o relacionados con el lenguaje receptivo. Otro factor que determina el éxito de la terapia logopédica es que su duración sea superior a 8 semanas.

\section{La familia como ayuda en la intervención.}

Desde esta aproximación, los terapeutas siguen siendo los encargados directos de la intervención sobre el niño. No obstante, los padres apoyan algunas fases de la intervención, realizando actividades concretas y asistiendo a la terapia con el niño. En algunos casos, el terapeuta da instrucciones y pautas a seguir a los padres sobre su propia conducta lingüística, y sobre cómo comportarse o responder al niño en casos determinados, pero sin un entrena- 
miento específico. Es frecuente que el terapeuta también establezca comunicación con otros entornos de la vida del niño, como el escolar. En definitiva, el papel fundamental de la familia en este tipo de intervención es garantizar el mantenimiento de los progresos adquiridos en el contexto de la terapia, y su generalización al contexto familiar (Girolametto y Weitzman, 2008).

A pesar de su participación en el proceso de intervención, el papel de los padres en este modelo de trabajo es difícil de operativizar, por lo que no existen suficientes estudios que prueben el efecto concreto que produce en el rendimiento lingüístico del niño.

La aproximación interaccionista en los trastornos del lenguaje: La intervención centrada en la familia.

Las teorías sociointeraccionistas conceden un papel relevante al adulto en el proceso de construcción del conocimiento del niño (Vigotsky, 1986). Así, en el caso del lenguaje, se entiende que su desarrollo se encuentra mediatizado por el papel que juegan los adultos significativos para el niño, que son capaces de ajustarse a sus limitaciones, y de atribuir significado a sus conductas cuando aún no existe intención en el acto del niño (Tomasello, 1988). En el marco de esta comunicación prelingüística, multitud de estudios han probado que el grado de sensibilidad y ajuste que muestran los cuidadores está directamente relacionado con el momento en que aparece el lenguaje, y con el grado de competencia lingüística que muestra el niño en los años posteriores (Hwa-Froellch, Loveland Cook y Frick, 2008; Pressman, PippSiegel, Yoshinaga-Itano y Deas, 1999). Una vez que aparece propiamente el lenguaje, el papel de los padres se ha mostrado también un potente predictor de las habilidades lingüísticas del niño (Silven, Niemi y Voeten, 2002). De hecho, existe evidencia de que una de las variables que influye de forma más determinante sobre la aparición del trastorno del lenguaje, es el uso de un "input inadecuado y deficitario por parte de los padres" (Acosta y Moreno, 1999). En concreto, se ha constatado el efecto de algunos factores como la "responsividad" de los padres a las vocalizaciones del niño, la reciprocidad y frecuencia en la interacción verbal, y la disponibilidad de materiales estimulantes (Peterson, 2004). En consecuencia, la conducta de los padres cobra especial relevancia en el proceso de mejora y tratamiento del trastorno del lenguaje del niño. 
Desde esta perspectiva, el proceso de intervención de los trastornos del lenguaje necesariamente considera a la familia como miembro activo del equipo interdisciplinar, (Grácia, 2002). No obstante, el grado de implicación varía mucho en función del planteamiento de la intervención. Mientras que algunas terapias combinan el trabajo de los padres con el trabajo de otros profesionales (Garcia y Turk, 2007; Gillette, 1992; Greenburg, 1992; Iacono, Chan y Waring, 1998), otras conceden a la familia el peso total y único del tratamiento (Girolametto, Weitzman y Clements-Baartman, 1998; Hemmeter y Kaiser, 1994).

\section{La aproximación interaccionista de la intervención en los trastornos del lenguaje: aspec- tos conceptuales.}

Los enfoques actuales de la intervención en niños desde la perspectiva interaccionista abogan por una detección y atención temprana de los trastornos, lo que supone implicar en mayor medida a las familias, puesto que los niños pequeños se adaptan peor a las terapias con adultos desconocidos. De hecho, estudios previos reconocen que cuanto más pequeño es el niño, mayor flexibilidad debe tener la terapia para tener éxito, y mayor presencia deben tener los padres en el proceso de intervención (Bryson, Rogers y Fombonne, 2003; Pretti-Frontczak y Bricker, 2004). Debido a estos hallazgos, algunos países como EEUU han comenzado a legislar la implicación paterna en la intervención temprana y en los contextos naturales, con el fin de mejorar el rendimiento de los niños, abaratar costes provenientes de servicios externos, y mejorar el grado de satisfacción de las familias (Individuals with Disabilities Education Act "IDEA"; en Girolametto y Weitzman, 2008).

Además, en las terapias centradas en familia también existen grandes variaciones en el tipo de entrenamiento que reciben los padres (Lederer y Kogan, 2007). Así, hay programas que buscan cambiar el "ambiente social" del niño (ej. Tannock, Girolametto y Siegel, 1992), y enfocan las conductas de los padres no sólo al cambio en pautas lingüísticas, (como el número de expansiones, requerimiento, o referentes que utilizan) sino también al cambio en pautas socio-afectivas (sensibilidad del cuidador ante las señales del niño, grado de interactividad mostrado en la interacción, o ambiente emocional del hogar). Desde esta aproximación, se considera que una base socio-emocional sólida es necesaria y beneficiosa para una adecuada asimilación de los aprendizajes. Por otro lado, otros programas se centran más en el "ambiente físico" del niño. Desde esta aproximación, se plantea el entrenamiento a los padres en 
la provisión de ambientes físicos adecuados, donde los juguetes y las actividades son altamente motivantes para el niño, consiguiendo así más y mejores oportunidades para el aprendizaje (ej. Dunst, 2001).

A pesar de que todos los programas centrados en la familia comparten la necesidad de dar a ésta un mayor protagonismo en el proceso de intervención, a lo largo de los años han variado las estrategias y el planteamiento teórico desde el que se implementaba el plan de intervención con padres.

Durante los años 60 comienzan a aparecer los primeros programas de intervención que incluyen a los padres como agentes activos de la terapia, fundamentalmente orientados al tratamiento en niños sordos, aunque no existen trabajos empíricos que constaten su eficacia (Grácia, 2002).

En los años 70, no obstante, es cuando se observa un aumento de la aplicación de este tipo de programas, aunque aún existen pocos estudios que los evalúen. Estos programas varían en el tipo de técnicas en las que se entrena a los padres (Girolametto y Weitzman, 2008). Por un lado, hay programas que inciden en el aprendizaje por parte de los padres de técnicas conductistas de refuerzo y castigo de la conducta, a través de las cuales se consiga el modelado del lenguaje del niño, como el programa ELI (McDonald y Preser Blott, 1974). Por otro lado, los programas de intervención basados en la perspectiva sociointeraccionista abogan por intervenciones en el contexto natural, en las que los padres respondan adaptándose al nivel del niño, sin el empleo de estrategias tan rígidas como las conductictas (Cheseldine y McConkey, 1979; Seitz, 1975). Aunque los programas aplicados desde ambas perspectivas parecen aumentar el rendimiento lingüístico del niño, se observa en ambos deficiencias metodológicas importantes que imposibilitan establecer relación causal entre la ganancia lingüística y la acción de los padres (Girolametto y Weitzman, 2008).

Es en la década posterior cuando se produce un auténtico interés por la evaluación de este tipo de programas, además de una mayor proliferación de los mismos. Durante estos años parece afianzarse la perspectiva sociointeraccionista de la intervención, en concreto la aproximación naturalista, por lo que la mayor parte de los programas se plantean en el contexto del hogar, y son los padres los que aplican la intervención una vez entrenados por el terapeuta. Es el caso de los programas Putting Two Words Together (McConkey y O Connor, 
1982), Language Interaction Intervention Project (Weitstuch y Lewis, 1985; Weistuch y Brown, 1987), o Transactional Intervention Program (Mahoney y Powell, 1988). Mención aparte merece por el alcance de su influencia el Programa Hanen (It Takes Two to Talk; Manolson, 1985), que aparece en estos años pero que se desarrolla y perfecciona en las décadas siguientes (Baxendale y Hesketh, 2003; Girolametto, Pearce y Weitzman, 1996; Girolametto, Tannock y Siegel, 1993; Tannock, Girolametto y Siegel, 1992), y que se ha aplicado en multitud de países y traducido a varios idiomas. Sólo unos pocos de los programas relevantes de estos años se mantiene fiel a la tradición conductista, insistiendo en el entrenamiento en técnicas de refuerzo y modelado de la conducta, como son el Milieu Teaching (Warren y Kaiser, 1986), o el Ecological Communication Organization Program (McDonald, 1989), aunque ambos programas se plantean en contextos naturales de interacción. Respecto a los resultados obtenidos tras la aplicación de estos programas, los estudios empíricos reconocen efectos positivos en el rendimiento lingüístico del niño posterior a la intervención centrada en la familia, aunque aún se detectan errores importantes fruto de falta de rigor metodológico.

Muchos de los programas nacidos en los 80 se perfeccionan y siguen aplicando en los años posteriores. Es el caso del Enhanced Milieu Teaching (Kaiser y Hester, 1994) o el Responsivity Education and Prelingüistic Milieu Teaching (Yoder y Warren, 2002), basados en el Milieu Teaching (Warren y Kaiser, 1986); el programa It Takes Two to Talk (Baxendale y Hesketh, 2003; Girolametto, Pearce y Weitzman, 1996; Girolametto, Tannock y Siegel, 1993; Tannock, Girolametto y Siegel, 1992), basado en el programa original de Manolson (1985); los programas Relationship-focused Intervention Program (Mahoney, Wiggers y Lash, 1996) y Responsive Teaching (Mahoney y Perales, 2005), basados en el Transactional Intervention Program (Mahoney y Powell, 1988); o el programa Ecological Communication Organization Program (McDonald y Wilkening, 1994), basado en el original de McDonald. Otros se crean en estos años, como el Focused Stimulation (Fey, Cleave, Long y Hughes, 1993) o el Parent Group Language Intervention (Gibbard, 1994), entre otros.

En las dos últimas décadas se observan tres tendencias significativas en el estudio de los programas de intervención con padres (Girolametto y Weitzman, 2008). Por un lado, se busca el aumento en la naturalidad de las estrategias utilizadas en la intervención logopédica, y un incremento en el entrenamiento a padres en sensibilidad y receptividad a las señales del niño. Desde este punto de vista, se plantea la mejora en las relaciones afectivas y sociales de los padres con sus hijos como base para el adecuado aprendizaje de nuevas habilidades por 
parte del niño (Niccols y Mohamed, 2000). Además, se observa un mayor rigor metodológico en la aplicación y evaluación de los programas, lo que permite establecer más fácilmente relaciones causales entre la ganancia lingüística del niño y la intervención familiar (Girolametto y Weitzman, 2008). Por último, multitud de estudios se plantean cuestiones sobre la eficacia de este tipo de terapia. De éstos, sólo unos pocos estudios han buscado comparar la eficacia de los programas centrados en la familia y los programas aplicados desde la terapia tradicional. A continuación detallamos los resultados de algunos de estos estudios.

\section{Eficacia de la terapia interaccionista en los trastornos del lenguaje en niños: Estudios empíricos.}

Como hemos comentado en el apartado anterior, en los últimos años se produce un aumento de estudios empíricos que buscan probar la eficacia de la terapia interaccionista en la intervención de los trastornos del lenguaje. En concreto, la mayor parte de los estudios miden la eficacia de la intervención familiar comparando su efecto con grupos que no han recibido tratamiento. Son muy pocos los estudios que han comparado el rendimiento de grupos en los que se ha aplicado intervención tradicional y sociointeraccionista. Los resultados de ambos grupos de trabajos se exponen a continuación, y aparecen también de manera resumida en el Anexo 1.

Eficacia de la terapia interaccionista comparada con grupos que no han recibido tratamiento.

Cuando hablamos de trastornos del lenguaje, vamos a dividir los estudios en aquellos referidos a trastornos específicos del lenguaje, en sus componentes fonológico, semántico, morfosintáctico y pragmático; y los trastornos del lenguaje asociados a otras patologías. Esta distinción no se basa en ninguna categorización clínica, sino que está motivada por la búsqueda de coherencia y claridad en la exposición de los resultados encontrados.

En cuanto al trastorno específico del lenguaje (TEL) y siguiendo la propuesta de la ASHA (American Speech-Hearing Association), entendemos que consiste en "una dificultad con el lenguaje que no está causada por ningún déficit evidente a nivel neurológico, sensorial, intelectual o emocional, y que puede afectar al desarrollo del vocabulario, la gramática y las habilidades conversacionales" (Aguado, 2006; Erven, 2001). 
Dentro los trastorno específicos del lenguaje es importante tener en cuenta que la diferenciación de cada uno de los componentes del sistema lingüístico es artificial, pues en la práctica se presentan de forma interrelacionada. No obstante esta distinción nos ayuda a agrupar de forma más coherente los trastornos y los programas de intervención, que establecen pautas de actuación distintas en función del área lingüística afectada (Acosta y Moreno, 1999).

Por tanto, consideramos en primer lugar el componente fonológico y fonético, que "incluye el estudio de los sonidos de la expresión lingüística desde un punto de vista funcional y abstracto, por un lado, y el estudio del material sonoro desde tres puntos de vista; de la producción, de su transmisión y de la percepción" (Acosta y Moreno, 1999). La terapia centrada en familia en los niños afectados por el componente fonológico cobra especial importancia dado el alto grado de "heredabilidad" que presentan estos trastornos. Uno de los programas más importantes en la terapia fonológica aplicada a familias de niños pre-escolares es el Parents and Children Together, desarrollado en Australia, y cuyo modelo de trabajo se basa en la educación familiar (técnicas conductuales y lingüísticas), tareas metaanalíticas (razonamiento sobre la propia fonología), entrenamiento en producción fonética, aprendizaje basado en ejemplos y trabajo en el hogar (Bowen y Cupples, 1999). En un estudio posterior, sus autores han probado la eficacia del programa, obteniendo mejoras significativas en el grupo que recibió terapia, comparado con el control (Bowen y Cupples, 2004).

En el caso de la tartamudez, se han aplicado entre otros el Southwest Texas Program, que incluye en la terapia a los padres y hermanos mayores de 6 años, y cuya eficacia ha sido evaluada en un estudio de Mallard (1998). Según este estudio, el entrenamiento en resolución de problemas y habilidades sociales por parte de los miembros de la familia consiguió en un $82 \%$ de los niños una mejora en el habla, y, como consecuencia, un decremento en la asistencia a la terapia logopédica. Otro estudio que prueba la mejora en tartamudez tras la intervención familiar es el de Mathews, Williams y Pring (1997). En el programa evaluado en este estudio los padres eran entrenados en habilidades de fluencia, y posteriormente aplicaban las técnicas aprendidas en la actividad diaria con sus hijos. La mejora encontrada en los niños se mantuvo hasta varias semanas después de la terapia. Un estudio reciente de Millard, Nicholas y Cook (2008) constata también la mejoría en el patrón de tartamudeo del niño de entre 3 y 4 años tras un año de terapia basada en el entrenamiento en pautas de interacción a los padres de niños afectados. 
Por otro lado, el programa Focused Stimulation (Fey, Cleave, Long y Hughes, 1993), en principio diseñado para la intervención léxica de los niños con retraso en el vocabulario, parece incrementar también el rendimiento en el área fonológica. Así, tras la intervención, dos medidas de diversidad fonológica (nivel de estructura silábica e inventario consonántico) mostraron mejoría comparados con el grupo que no había recibido intervención (Girolametto, Pearce y Weitzman, 1997).

El componente semántico se refiere "al estudio del significado de los signos lingüísticos y de sus posibles combinaciones en los diferentes niveles de organización del sistema lingüístico" (Acosta y Moreno, 1999). En relación a este componente, la mayor parte de los estudios se han centrado en el análisis de la competencia léxica, en concreto en los deterioros encontrados en el lenguaje expresivo o receptivo. En este área, el programa Hanen (It Takes Two to Talk; Manolson, 1985) ha mostrado grandes niveles de eficacia, siendo muchos los estudios que han probado un aumento en el vocabulario y la producción y comprensión lingüística de los niños tras su implementación (Girolametto, Pearce y Weitzman, 1996; Moller, Probst y Hess, 2008). No obstante, el programa Hanen no ha sido evaluado únicamente en cuanto a ganancia lingüística de los niños, sino también en cuanto a cambios conductuales de los padres tras el tratamiento. Por un lado, se han encontrado variaciones generales en las forma de hablar de las madres tras el entrenamiento. En concreto, la forma de hablar es más lenta, con un vocabulario menos complejo y más focalizado al interés del niño. Por otro lado, se han encontrado un mayor uso de estrategias centradas en el niño, promotoras de la interacción y modeladoras del lenguaje en los padres tras el entrenamiento en este tipo de programa. (Girolametto y Weitzman, 2008).

Otros programas que tienen en cuenta el estilo de vida familiar también se han mostrado eficaces en el aumento de vocabulario en niños con retrasos del lenguaje expresivo. En concreto, el programa CBLA (Chile Health Status Assessment; Chao, Bryan, Burstein y Ergul, 2006) incluye actividades promotoras del lenguaje que se insertan en actividades de la vida diaria como son comer, ver la tele o comprar, y se ha mostrado altamente eficaz en la mejora de las alteraciones del lenguaje expresivo. El programa Parent Group Language Intervention (Gibbard, 1994), con niños entre 2 y 3 años, mostró ganancias significativas en lenguaje expresivo de los niños tras el entrenamiento en habilidades lingüísticas de sus padres. Uno de los programas que también se ha mostrado eficaz para niños que hablan tarde es el Focused Stimulation (Fey, Cleave, Long y Hughes, 1993), un tratamiento que entrena a los 
padres en aumento de su sensibilidad y receptividad a las señales del niño, y que busca proporcionar modelos lingüísticos ajustados al nivel del niño. En un estudio sobre este programa de Pearce, Girolametto y Weitzman (1996) se constató un aumento en el nivel de vocabulario del niño, y un cambio en la conducta materna en las situaciones de interacción. En concreto, se observa una disminución en la complejidad del habla materna, un aumento de la referencia a objetos, y una disminución en sus patrones de directividad. Otro estudio de Lederer (2001), también prueba la eficacia de este programa en el nivel de vocabulario alcanzado por el niño tras la intervención. Más recientemente, el Programa Heidelberg Parent-based Language Intervention también se mostró eficaz en relación a las alteraciones relacionadas con el lenguaje expresivo. Así, un estudio de Buschmann, Jooss, Rupp, Fedhusen, Pietz y Philippi (2009) constató que tras su aplicación en niños de 2 años, un $75 \%$ de niños con 3 años mostraron niveles normales de habilidades expresivas, comparadas con un $44 \%$ de los niños que tuvieron estas mismas puntuaciones y que no recibieron tratamiento. Por otro lado, el programa Enhanced Milieu Teaching (Hemmeter y Kaiser, 1994), ha probado un aumento en el vocabulario receptivo del niño tras su aplicación (Kaiser y Hancock, 2003).

El componente morfosintáctico hace referencia "al componente lingüístico que se ocupa del estudio de las reglas que intervienen en la formación de las palabras y de las posibles combinaciones de éstas en el interior de las diferentes secuencias oracionales en las que se estructura una lengua" (Acosta y Moreno, 1999). Existen pocos estudios que hayan evaluado la mejora en las habilidades morfosintácticas en el niño tras el entrenamiento específico de los padres. Al igual que ocurría con el componente fonológico y semántico, el programa Focused Stimulation (Fey, Cleave, Long y Hughes, 1993), se ha mostrado eficaz en el tratamiento de dificultades morfosintácticas. En concreto, se ha observado tras su implementación una ganancia sintáctica significativa medida a través de la escala DSS (Developmental Scale Score), que mide la diferencia en rendimiento de un curso a otro. El programa Enhanced Milieu Teaching (Hemmeter y Kaiser, 1994) también ha resultado útil en este tipo de dificultades, con un aumento de la producción sintáctica del niño tras su implementación. En este programa se parte de los intereses del niño y de los contextos naturales de interacción para promover estrategias facilitadoras del lenguaje. Además, utiliza las técnicas planteadas desde el Milieu Teaching (Intervención en contextos de producción; en Acosta y Moreno, 1999), como son el modelaje de la conducta verbal a través de ejemplos y apoyos específicos, vinculados a la interacción padre-hijo. Desde este programa se plantea también la denominada "enseñanza incidental", que plantea la enseñanza en contextos naturales no estructurados como el juego 
libre, y que parte de las iniciativas del niño con el fin de mantener su motivación e interés. Varios trabajos han constatado el empleo de este de este programa con éxito (Kaiser, Hancock y Trent, 2007; Kaiser y Hester, 1994; Peterson, Carta y Greenwood, 2005).

Por último, el componente pragmático hace referencia al estudio del lenguaje en contextos sociales (Acosta y Moreno, 1999). En este sentido, nuevamente el programa Hanen (It Takes Two to Talk; Manolson, 1985) resulta útil en el aumento de las habilidades conversacionales del niño, como son la toma de turnos o el mantenimiento de un tema de conversación (Coulter y Gallhager, 2001). Otros programas eficaces en esta área son el Ecological Communication Organization Program (ECO; McDonald y Wilkening, 1994), aplicado en niños de entre 2 y 4 años, y que se centra en la creación de rutinas de conversación con el objetivo de que el niño aprenda a conversar con independencia del contexto en el que esté; y el Responsivity Education and Prelinguistic Milieu Teaching (Yoder y Warren, 2002), una variación del Programa Mielieu Teaching original. Basada en la filosofía de este programa se planteó la intervención de 4 niños de entre 24 y 31 meses, con retrasos en lenguaje expresivo, receptivo, y habilidades socio-lingüísticas (Mobayed, Collins, Stranggis, Schuster y Hemmeter, 2000). En concreto se entrenó a los padres en técnicas de "enseñanza” incidental” para instruir a sus hijos en la habilidad de pedir o solicitar algo. Estas técnicas se mostraron eficaces en su objetivo, de manera que los niños aprendieron como requerir algo y lo generalizaron a otros contextos, además de mantener estos logros un tiempo después de la intervención. Otro estudio sobre el entrenamiento a padres en el contexto de contar cuentos demostró la eficacia de este tipo de intervención, pues los niños incrementaron sus habilidades en la toma de turnos, y mostraron una mayor frecuencia de inicio de actos comunicativos (Crowe, Norris y Hoffman, 2000). Por último, un estudio de Bernhardt, Smith y Smith (1992) informó de mejoras en las habilidades comunicativas de los niños tras un programa colaborativo, en el que los niños recibían tanto terapia logópedica directa a través del logopeda, como indirecta a través de padres previamente entrenados.

Dentro de los trastornos del lenguaje asociados a otras patologías, el autismo ha sido uno de los más estudiados en las terapias interaccionistas. Son muchos los programas que han entrenado a padres para obtener mejoras lingüísticas en el niño autista, sobre todo en el área de la comunicación prevebal. Por ello muchos de estos programas han buscado entrenar a los padres en habilidades relacionadas con situaciones de atención conjunta, pues es en este contextos de atención compartida donde los niños autistas muestran mayores alteraciones, y 
los que a su vez se han mostrado precursores de la comunicación lingüística posterior (Siller y Sigman, 2002). Además, se han observado cambios colaterales positivos en el área social y del lenguaje cuando mejora la atención conjunta. En un estudio de Rocha, Schreibonan, y Stahmer (2007) se comprobó el aumento de conductas de atención conjunta en padres tras varias sesiones de entrenamiento específico, y un incremento consecuente en las respuestas de inicio y respuesta de atención conjunta por parte de los niños. Además, este estudio probó el mantenimiento de estas conductas en el período de seguimiento, además de altos grados de satisfacción parental. Resultados similares se han obtenido en otros estudios, en relación a la frecuencia de episodios de inicio y respuesta de atención conjunta, y al mantenimiento de estos resultados (Ingersoll y Dvortcsak, 2006; Schertz y Odom, 2007).

Otros programas aplicados a familias de niños autistas han incluido otro tipo de variables, además de la atención conjunta. Dado que estudios previos han encontrado mayor grado de directividad y control en padres de niños autistas, comparados con padres de niños normales, algunos programas han buscado entrenar a los padres en sus estilos de interacción. En un estudio de Mahoney y Perales (2003), se aplicó un programa en el que las madres eran entrenadas en estrategias de interacción "sensibles" (en conexión con las señales del niño). Los resultados de este estudio probaron un aumento en el uso de estas estrategias en un $80 \%$ de las madres, y un aumento en las puntuaciones de atención, persistencia, e inicio de episodios de atención conjunta por parte del niño. De hecho, las madres que más incrementaron el uso de estrategias interactivas sensibles fueron aquellas cuyos hijos obtuvieron las mayores ganancias socio-lingüísticas. Por otro lado, el programa Social Communication Intervention (Aldred, Pollard y Adams, 2001) entrena a los padres en estrategias a utilizar en las rutinas diarias, y cuyo objetivo es interpretar las señales del niño para una adaptación adecuada al mismo. La aplicación de este programa se ha mostrado eficaz, puesto que el grupo experimental que se sometió al tratamiento obtuvo mayores puntuaciones en vocabulario expresivo, además de mayor frecuencia de actos comunicativos, comparados con el grupo control (Aldred, Green y Adams, 2004).

Existe una variación del programa Hanen aplicado a familias de niños autistas (More than words; Sussman, 1999). Varios estudios han probado su eficacia en el incremento de vocabulario medido en los niños a través del CDI (MacArthur-Bates Communicative Developmental Inventory; Fenson, Dale, Reznick, Thal, Bates, y Hartung (2003), además de un aumento de estrategias óptimas de interacción por parte de los padres (Aldred et al., 2004; 
McConachie, Randle, Hammal y Le Couteur, 2005). En un estudio más completo, se ha probado la eficacia de este programa en el aumento de estrategias de interacción responsivas por parte de los padres, el aumento de las puntuaciones en vocabulario, medido a través del CDI y en una interacción grabada de juego libre, y una mejora significativas de las habilidades sociales de interacción por parte del niño (Girolametto, Sussman y Weitzman, 2007).

Otra de las patologías que se asocia a trastornos en el lenguaje es la presencia de retrasos cognitivos en el niño. Una de las más estudiadas ha sido el Síndrome de Down. En este sentido, estudios del equipo de Vilaseca (2004) prueban que las madres de niños con deficiencia utilizan de forma espontánea estrategias poco óptimas en el desarrollo del lenguaje morfosintáctico de estos niños, y sugieren el entrenamiento en una serie de estrategias facilitadoras en el contexto natural. Uno de los primeros programas aplicados a padres para niños con este retraso fue el de Chelsedine y McConkey (1979), que probó la eficacia de la intervención, y señaló el mayor apoyo que necesitan los padres sobre las madres, en cuanto a entrenamiento en estrategias de comunicación. Por otro lado, se ha probado con éxito el programa de intervención Focused Stimulation (Fey, Cleave, Long y Hughes, 1993), observándose un aumento en el vocabulario mostrado por los niños, además de una mayor frecuencia de uso de estrategias facilitadotas de lenguaje por parte de los padres (Girolametto, Weitzman y Clements-Baartman, 1998). Un estudio que probó un aumento en las habilidades conversacionales de niños con Síndrome de Down fue el de Trent-Strainbook, Kaiser y Frey (2007), que incluye a los hermanos mayores como "modeladores" del lenguaje de los niños. Otros programas aplicados con éxito en el aumento de vocabulario, la combinación de palabras y la densidad léxica en niños con retraso mental son el Putting Two Words Together (Cheseldine y McConkeym, 1979) y el Responsivity Education and Prelinguistic Milieu Teaching (Yoder y Warren, 2002). La aplicación del programa Hanen en niños con retraso cognitivo ha probado también un incremento en habilidades conversacionales, vocabulario y productividad lingüística (Girolametto, 1988; Tannock, Girolametto y Siegel, 1992).

Por último, un estudio de Pennington, Golbart y Marshall (2004) analizó la eficacia de varios programas en los que se entrenaba a padres en habilidades conversacionales a niños con parálisis cerebral. En concreto, el objetivo de estos programas es aumentar la sensibilidad de los padres a los intentos comunicativos del niño, y enseñarles a crear oportunidades donde los niños puedan tomar un rol más independiente y equitativo en la conversación. Las conclusiones de este estudio señalan la eficacia de estas técnicas, a pesar de los fallos metodológicos 
encontrados en muchos de estos estudios. En general, existen datos empíricos concluyentes sobre el beneficio de la inclusión de padres en intervención con niños con retrasos cognitivos, independientemente de los resultados lingüísticos del niño, dado el decremento en los niveles de estrés, ansiedad y depresión observados en padres tras el entrenamiento y la intervención (Pelchat, Bisson, Ricard, Perreault y Bouchard, 1999).

Por último, la sordera e hipoacusia son patologías que afectan profundamente al desarrollo del lenguaje por parte del niño. Además, la aplicación del tratamiento pasa por el aprendizaje por parte de padres de nuevas formas de comunicación, lo que incrementa los niveles de estrés que vive la familia durante el proceso. No obstante, tal como ocurre en otras patologías, las familias que participan activamente en la intervención lingüística del niño sordo obtienen mejores puntuaciones tanto en vocabulario, como en razonamiento verbal de sus hijos (Moeller, 2000). Además, en un estudio de Zaidman-Zait y Young (2007) se demuestra que el entrenamiento a padres dirigido a objetivos basado en la teoría de la acción favorece el proceso de habilitación del niño posterior a un implante coclear. Esta intervención parte de los hallazgos de DesJardin (2004), según los cuales mayores niveles de implicación materna correlacionan positivamente con un mayor uso de estrategias facilitadoras del lenguaje en niños con problemas de audición. Además, Kargin (2004) también ha probado la eficacia de la intervención interaccionista lingüística en niños sordos de entre 0 y 4 años, comparados con grupos control. Otros programas para padres de niños sordos que se han aplicado con éxito son el Bill Wilkerson Hearing and Speech Center Program (McConnell, 1974) el Programa Webster-Straton modificado (García y Turk, 2007) y el programa The Counseling and Home Training Program (Greenburg, 1982). Un estudio de Harrigan y Nikolopoulos (2002) encontró que el entrenamiento a padres de niños sordos mejoró sus estrategias de gestión de la comunicación, ya que se observó un decremento en directividad, y un menor inicio de actos comunicativos. Por último, existe una adaptación del programa Hanen para niños sordos (Manolson, 1992), cuya eficacia ha sido probada en estudios experimentales en niños sordos con implante coclear. Así, se han observado mejoras en las habilidades comunicativas del niño sordo, además de un aumento significativo de su vocabulario (Girolametto, 1988; Tannock, Girolametto y Siegel, 1992).

Para terminar, cabe mencionar que las terapias interaccionistas que incluyen a la familia no solo han mostrado su eficacia en el rendimiento lingüístico de padres e hijos, sino también en otro tipo de variables que afectan a la dinámica familiar. Así, tras el entrenamiento y 
participación de los padres en el proceso de intervención se han encontrado, entre otros; mayores niveles de satisfacción con los resultados de la intervención (Applequist y Bailey, 2000; Lederer, 2001; Niccols y Mohamed, 2000), una disminución de los niveles de estrés, depresión y ansiedad (Greengurg, 1982; Klasen, 1995; Niccols y Mohamed, 2000), mejor ajuste emocional con los niños (Hemmeter y Kaiser, 1994; Klasen, 1995) y sentimientos de mayor competencia y eficacia por parte de todos los miembros de la familia (Lederer, 2001; Trivette, Dunst, Boyd y Hamby, 1996).

\section{Eficacia de la terapia interaccionista comparada con la terapia tradicional.}

Aunque la eficacia de las terapias centradas en la familia es incuestionable, a la luz de los resultados descritos en el apartado anterior, aún son pocos los estudios que han buscado comparar la eficacia de este tipo de terapias con la intervención centrada en el logopeda.

En este sentido, la mayoría de estudios han encontrado, cuanto menos, resultados similares en ambos tipos de terapia. Así, en el estudio de Baxendale y Hesketh (2003), se administró ambos tipos de terapia en dos grupos, y en un $71 \%$ de los niños se dieron ganancias lingüísticas significativas, aunque no hubo diferencias significativas cuando se compararon ambos grupos. Este estudio prueba el hecho de que los programas interaccionistas son más intensos, en términos de tiempo de terapia, por lo que se necesitan más recursos para su puesta en marcha. En una revisión de varios estudios realizada por Law, Garrett y Nye (2004) tampoco se encontraron diferencias significativas en el rendimiento de los niños tras la intervención dirigida por el logopeda, comparada con la dirigida por padres entrenados. Otros estudios, como el de Fey et al. (1993), concede cierta ventaja en el mantenimiento y consistencia de los resultados en la terapia tradicional, mientras que Gibbard, Coglan y Mcdonald (1994; 2004) encuentran mayor rendimiento lingüístico en niños que participan en terapias interaccionistas, comparados con los que reciben únicamente tratamiento logopédico.

\section{Conclusiones.}

Esta revisión teórica pone de manifiesto la importancia de la familia en relación al desarrollo del lenguaje y sus posibles trastornos. Teniendo en cuenta que es en el contexto familiar donde el niño aprende y desarrolla sus habilidades lingüísticas, y dada la evidencia empí- 
rica que apoya la intervención temprana de los trastornos del lenguaje, se hace difícil plantear un programa de intervención que obvie el papel de los padres. Aunque la terapia estrictamente logopédica se muestra útil en la evolución y mejora de los trastornos del lenguaje, la implicación familiar en el proceso de intervención no solo aumenta el rendimiento lingüístico del niño, sino que favorece el uso de contextos naturales donde la generalización por parte del niño es más fácil y perdurable. Además, al tener en cuenta a la familia, no sólo se mejora el input lingüístico del niño y sus padres, sino que se estrechan los vínculos afectivos y aumenta el grado de satisfacción mostrado por todos los miembros de la familia. Se suaviza así el impacto negativo de la crianza de un niño con retraso evolutivo lingüístico. Los estudios empíricos demuestran estos efectos positivos sobre las áreas social, emocional y del lenguaje.

No obstante, los estudios que han analizado las terapias que incluyen a la familia también han sacado a la luz ciertas limitaciones. En concreto, se hace difícil en muchos estudios establecer relaciones causales entre las ganancias lingüísticas observadas en los niños, y la acción directa de los padres sobre éstas. Se hacen necesarios por tanto, estudios que cuiden el rigor metodológico y aseguren la causalidad de esta relación (Girolametto y Weitman, 2008). Además, muchos estudios plantean que algunos programas supuestamente centrados en la familia siguen siendo estrictamente controlados por el logopeda y sitúan la actuación paterna a un segundo orden (Brown y Nott, 2006). Otros hacen alusión a la escasa coordinación entre los servicios externos que plantean la intervención y las familias objeto de la misma (Bayley, 2001; Romer y Umbreit, 1998). Por último, se hacen necesarios más estudios de seguimiento que garanticen los efectos positivos de la terapia familiar pasado un tiempo de la intervención, pues la mayoría de los estudios se han centrado en los efectos sobre el niño durante la intervención o muy pocas semanas después de ésta.

\section{Referencias}

Acosta, V. M. y Moreno, A. M. (1999). Dificultades del lenguaje en ambientes educativos. Barcelona: Masson.

Acosta, V.M. (2004). Las prácticas educativas ante las dificultades del lenguaje. Una propuesta desde la acción. Barcelona: Ars Médica.

Aguado, G. (2006). Dificultades en el lenguaje: Trastorno Específico del Lenguaje. En J. L. Gallego (Dir.). Enciclopedia temática de Logopedia (pp 17-44). Antequera: Aljibe. 
Aldred, C., Pollard, C., y Adams, C. (2001). Child'sTalk: for children with autism and pervasive developmental disorder. International Journal Language Communication Disorder, 36, pp. 46974.

Aldred, C.R., Green, J. y Adams, C. (2004) A new social communication intervention for children with autism: pilot randomised controlled treatment study suggesting effectiveness. Journal of Child Psychology and Psychiatry, 45:, pp. 1-11

Applequist, K. L., y Bailey, D. B. (2000). Navajo Caregivers' Perceptions of Early Intervention Services. Journal of Early Intervention, 23(1),pp. 47-61.

Baxendale, J., y Hesketh, A. (2003). Comparison of the effectiveness of the Hanen Parent Programme and traditional clinic therapy. International Journal of Language and Communication Disorders, 38, pp.397-415.

Bayley, D. B. (2001). Evaluating parent involvement and family support in early intervention and preschool programs. Journal of Early Intervention, 24(1);pp. 1-14.

Bernhardt, B., Smith, D., y Smith, R. (1992). Language intervention with a family-centered, collaborative, transdiciplinary, integrated approach: An example. Child Language Teaching and Therapy, 8; pp.265-284.

Bowen, C. y Cupples, L. (2004). The role of families in optimizing phonological therapy outcomes. Child Language Teaching and Therapy, 20(3), pp.245-260.

Brown, P. M. y Nott, P. (2006). Family-Centered Practice in Early Intervention for Oral Language Development: Philosophy, Methods, and Results. Spencer, Patricia Elizabeth (Ed); Marschark, Marc (Ed). (2006). Advances in the spoken language development of deaf and hard-of-hearing children. (pp. 136-165). xvii, 381 pp. New York, NY, US: Oxford University Press.

Bruner, J. (1983): El Habla del Niño. Ed. Paidós. Bs. As.

Bryson, S. E., Rogers, S. J., y Fombonne, E. (2003). Autism spectrum disorders: Early detection, intervention, Education, and psychopharmacological management. Canadian Journal of Psychiatry, 48, pp.506-516.

Buschmann, A., Jooss, B., Rupp, A., Fedhusen, F., Pietz, J., y Philippi, H.(2009). Parent based language intervention for 2-year-old children with specific expressive language delay: a randomised controlled trial. Archives Disabled Children, 94(2), pp.110-116.

Cheseldine, S. y McConkey R. (1979). Parental speech to young Down's syndrome children: an intervention study. American Journal of Mental Deficiency, 83, pp. 612-620.

Coulter, L. y Gallhager, C. (2001). Evaluation of the Hanen early childhood educators programme. International journal of language y communication disorders, 36 Suppl: pp. 264-9.

Crowe, L.K., Norris, J.A., y Hoffman, P.R. (2000). Facilitating storybook interactions between mothers and their preschoolers with language impairment. Communication Disorders Quarterly, 21(3) pp.131-146. 
DesJardin, J.L.(2004). Maternal Perceptions of Self-Efficacy and Involvement in the Auditory Development of Young Children with Prelingual Deafness. Journal of Early Intervention, 27(3), pp. 193-209

Dunst, C. J. (2002). Family-centered practices: Birth through high school. Journal of Special Education, 36, pp.139-147.

Erven, M. (2001). SLI: What we know and why it matters. The ASHA Leader Online.

Fenson, L., Bates, E., Dale, P., Goodman, J., Reznik, S., y Thal, D., (2000): Measuring variability in early child language: Don't shoot the messenger. Child Development, 71, pp. 323-328.

Fey, M., Cleave, P., Long, S., y Hughes, D. (1993).Two approaches to the facilitation of grammar in children with language impairment: An experimental evaluation. Journal of Speech and Hearing Research, 36, pp.141-157.

Garcia, R. y Turk, J. (2007). The Applicability of Webster-Stratton Parenting Programmes to Deaf Children with Emotional and Behavioural Problems, and Autism, and Their Families: Annotation and Case Report of a Child with Autistic Spectrum Disorder. Clinical Child Psychology and Psychiatry, 12, pp.125-136.

Gibbard, D. (1994). Parental-based intervention with pre-school language delayed children. European Journal of Disorders of Communication, 29, pp.131-150.

Gibbard, D., Coglan, L., y MacDonald, J. (2004). Cost-effectiveness analysis of current practice and parent intervention for children under 3 years presenting with expressive language delay. International Journal of Language and Communication Disorders, 39, pp. 229 - 244

Gillette, Y. (1992). Family-centered early intervention: an opportunity for creative practice in speechlanguage pathology. Clinics in communication disorders, 2(3); pp.48-60.

Girolametto, L. (1988). Improving the social-conversational skills of developmentally delayed children: An intervention study. Journal of Speech and Hearing Disorders, 53, pp.156-167.

Girolametto, L., y Weitzman, E. (2008). Working with families of young children with communication and language impairments: Intervention (p. 131-170). In N. Watts Pappas y S. McLeod (Eds). Working with families in speech-language pathology. San Diego, CA: Plural Publishing.

Girolametto, L., Pearce, P. y Weitzman, E. (1997). The Effects of Lexical Intervention On the Phonology of Late Talkers. Journal of Speech, Language and Hearing Research, 40, pp.338-348.

Girolametto, L., Sussman, F., y Weitzman, E. (2007). Using case study methods to investigate the effects of interactive intervention for children with Autism Spectrum Disorders. Journal of Communication Disorders, 40, pp.470-492.

Girolametto, L.,Tannock, R., y Siegel, L. (1993). Consumer-oriented evaluation of interactive language intervention. American Journal of Speech-LanguagePathology, 2, pp. 41-51. 
Girolametto, L.E., Pearce, P., y Weitzman, E. (1996). Interactive Focused Stimulation for Toddlers with Expressive Vocabulary Delays. Journal of Speech and Hearing Research, 39, pp.12741283.

Girolametto, L.E., Weitzman, E. y Clements-Baartman, J. (1998). Vocabulary Intervention for Children with Down Syndrome: Parent Training Using Focused Stimulation. Infant-Toddler Intervention, 8,pp. 109-125.

Goldstein, H., y Kaczmarek, L. (1992). Promoting communicative interaction among children in integrated intervention settings. In S. Warren y J. Reichle (Eds.), Causes and effects in communication and language intervention (pp. 81-111). Baltimore: Brookes.

Grácia, M. (2002). Comunicación y lenguaje en primeras edades: Intervención con familias. Editorial Milenio.

Greenburg, M.T. (1992). Families with Deaf Children: The Effects of Early Intervention. [Reports Research. Speeches/MeetingPapers].

Harrigan, S. y Nikolopoulos, T.P. (2002). Parent interaction course in order to enhance communication skills between parents and childrens following pediatric cochlear implantation. International Journal of Pediatric Otorhinolaryngology, 66, pp. 161-166.

Hemmeter, M.L., y Kaiser, A.P. (1994). Enhanced milieu teaching: Effects of parentimplemented language intervention. Journal of Early Intervention, 18, pp. 269-289.

Hwa-Froelich, D.A., Loveland Cook C.A., y Flick, L.H. (2008). Maternal Sensitivity and Communication Styles: Mothers With Depression. Journal of Early Intervention, 3(1); pp.44-66.

Iacono, TA, Chan JB, y Waring RE.(1998). Efficacy of a parent-implemented early language intervention based on collaborative consultation. Inernational Journal of Language Communication Disorders, 33: pp.281-303.

Ingersoll, B. y Dvortcsak, A (2006). Including parent training in the early childhood special education curriculum for children with autism spectrum disorders. Journal of Positive Behavior Interventions, 8 ,pp.79-87.

Kaiser, A. P., y Hancock, T. B. (2003). Teaching parents new skills to support their young children's development. Infants and Young Children, 16, pp.9-21.

Kaiser, A. P., y Hester, P. P. (1994). Generalized effects of enhanced milieu teaching. Journal of Speech and Hearing Research, 37, pp.1320-1340. 
Kaiser, A. P., Hancock, T. B., y Trent, J. A. (2007). Teaching parents communication strategies. Early Childhood Services: An Interdisciplinary Journal of Effectiveness, 1(2), pp.107-136.

Kargin, T. (2004). Effectiveness of a family-focused early intervention program in the education of children with hearing impairments living in rural areas. International Journal of Disability, Development, and Education, 51 (4), pp.401-418.

Klasin, K. (1995). Parent and child factors in individualizaded language intervention: An evaluation study. Dissertation Abstracts International Section A: Humanities and Social Sciences, vol.55 (2), p.3786.

Law, J. y Conti-Ramsden, G. (2000). Treating children with speech and language impairments. $B M J$, 321(7266), pp. 908-909.

Law, J., Garrett, Z., y Nye, C. (2004). The efficacy of treatment for children with developmental speech and language delay/disorder: a meta-analysis. Journal of speech, language, and hearing research, 47(4):pp. 924-43.

Lederer, S.H. (2001). Efficacy of parent-child language group intervention for late talking toddlers. Infant Toddler Intervention, 11(3), pp.223-235.

Lederer, S.H., y Kogan, E. (2007, 1). Collaborating with parents of young children with language delays. www.speechpathology.com, posted 1-22-07.

Mahoney, G., y Perales, F. (2005). Relationship-focused early intervention with children with pervasive developmental disorders and other disabilities: A comparative study. Journal of Developmental and Behavioral Pediatrics, 26, pp.77-85.

Mahoney, G., Wiggers, B., y Lash, S. (1996). Using a relationship-focused intervention program to enhance father involvement. Infant-Toddler Intervention, 6, pp.295-308.

Mahoney,G.,y Powell, A. (1988). Modifying parent-child interaction: Enhancing the development of handicapped children. Journal of Special Education, 22, pp.82-96.

Mallard, A. R. (1998). Using problem-solving procedures in family management of stuttering. Journal of Fluency Disorders, 23, pp.127-135.

Manolson, H. (1985). It takes two to talk: A parent's guide to helping children communicate (2nd ed.). Toronto, ON: The Hanen Centre.

Manolson, H. (1992). It takes two to talk: A parent's guide to helping children communicate (3rd ed.). Toronto, ON: The Hanen Centre.

Matthews S, Williams R y Pring T, (1997). Parent-child interaction therapy and dysfluency: a singlecase study. European Journal of Disorders of Communication 32, pp.346-357. 
McConachie, H, Randle, V, Hammal, D y Le Couteur, A. (2005). A controlled trial of training course for parents of children with suspected autism spectrum disorder. The Journal of Pediatrics 2005, 147(3), pp.335-340.

McConkey, R., y O’Connor, M. (1982). A new approach to parental involvement in language intervention programmes. Child: Care, Health and Development, 8, pp.163-176.

McConnell, F. (1974). The Parent Teaching Home: An Early Intervention Program for HearingImpaired Children Peabody. Journal of Education. 51, pp.162-70

McDonald, J., y Presser Blott, J. (1974). Environmental language intervention: The rationale for a diagnostic and training strategy through rules, context and generalization. Journal of Speech and Hearing Disorders, 39, pp.244-256.

McDonald, J., y Wilkening, P. (1994, May). Parent-child relationships for communicative development: An intervention study. Paper presented at the Annual Meeting of the American Association of Mental Retardation, Boston.

McDonald, J.D. (1989). Becoming partners with children. San Antonio, TX, Special Press.

Millard, SK, Nicholas, A y Cook, FM (2008). Is Parent-Child Interaction Therapy Effective in Reducing Stuttering?. Journal of Speech, Language and Hearing Research, 51(3), pp 636-650.

Mobayed, K. L., Collins, B. C., Schuster, J. W., Hemmeter, M. L., y Strangis, D. (2000). Teaching parents to employ mand-model procedures to teach their children to make requests. Journal of Early Intervention, 23, pp.165-179.

Moeller, M.P. (2000). Early intervention and language development in children who are deaf and hard of hearing. Pediatrics, 106(3); pp.1-9.

Moller, D., Probst, P., y Hess, M. (2008). Implementation and evaluation of a parent training for language delayed children. Praxis der Kinderpsychologie und Kinderpsychiatrie 57(3):pp.197215.

Nelson, K.E. (1977). Facilitating children's syntax acquisition. Developmental Psychology, 13, pp.109-117.

Niccols, A. y Mohammed, S. (2000). Parent Training in Groups: Pilot Study with Parents of Infants with Developmental Delay. Journal of Early Intervention, 23; pp.133-143.

Pearce, P.S., Girolametto, L.E., y Weitzman, E. (1996). Th effects of focused stimulation intervention on mothers of late-talking toddlers. Infant-Toddler intervention, 6, pp.213-227. 
Pelchat, D., Bisson, J., Ricard, N., Perreault, M., y Bouchard, J. (1999). Longitudinal effects of an early family intervention programme on the adaptation of parents of children with a disability. International Journal of Nursing Studies, 36, pp.465-477.

Pennington L, Goldbart J,y Marshall J. (2004). Interaction training for conversational partners of children with cerebral palsy: a systematic review. International Journal of Language and Communication Disorders, 39(2), pp.151-170.

Peterson, P. (2004). Naturalistic language teaching procedures for children at risk for language delays. The Behavior Analyst Today, 5(4), pp.404-424.

Peterson, P., Carta, J, y Greenwood, C. (2005). Teaching milieu language skills to parents in multiple risk families. Journal of Early Intervention, 27, pp.94-109.

Pressman, L., Pipp-Siegel, S., Yoshinaga-Itano, C. y Deas, A. (1999). The relation of sensitivity to child expressive language gain in deaf and hard-of-hearing children whose caregivers are hearing. The Journal of Deaf Studies and Deaf Education. 4(4), pp.294-304.

Pretti-Frontczak K.y Bricker, D.D. (2004). An activity based approach to early intervention (3 ${ }^{\mathrm{a}}$ Edic.). Baltimore: Paul Brookes Publishing Company.

Rocha, M.L., Schreibonan, L., y Stahmer, A.C. (2007). Effectiveness of training parents to teach joint attention in children with autism. Journal of Early Intervention, 29(2), pp.154-172.

Romer, E. F., y Umbreit, J. (1998). The effects of family-centered service coordination: A social validity study. Journal of Early Intervention, 21,pp. 95-110.

Schertz, H. y Odom, S. L. (2007). Promoting joint attention in toddlers with autism: A parentmediated developmental model. Journal of Autism and Developmental Disorders, 37, pp.1562-1575.

Seitz, S. (1975). Language intervention changing the language environment of the retarded child.En R. Koch, F. de la Cruz y F. Menolascino, (Eds). Down Syndrome: Reasearch, prevention and management. Nueva York: Bruner/Mazel.

Siller, M. y Sigman, M. (2002). The behaviors of parents of children with autism predict the subsequent development of their children's communication. Journal of Autism and Developmental Disorders, 32, pp.77-89.

Silven, M., Niemi, P., y Voeten, M. (2002). Do maternal interaction and early language predict phonological awareness in 3-to-4 year olds? Cognitive development, 17(1), pp.1133-1155. 
Tallal, P., y Rice, M.L (1998).Evaluating new training programs for language impairment. ASHA, 40, pp.1-7.

Tannock, R., Girolametto, L.E. y Siegel, L. (1992). The Interactive model of Language Intervention: Evaluation of its Effectiveness for Pre-School-Aged Children with Developmental Delay. American Journal of Mental Retardation, 97, (2), pp.145-160.

Tomasello, M. (1988). The role of joint attention in early language development. Language Sciences, 11, pp.69-88.

Trent-Stainbrook, A., Kaiser, A.P., y Frey, J.R. (2007). Older siblings'use pf responsive interaction strategies and effects on their younger siblings with Down Syndrome. Journal of Early Intervention, 29(4);pp. 273-286.

Trivette, C.M., Dunst, C.J., Boyd, K., y Hamby, D. (1996). Family-oriented program models, helpGiving practices, and parental control appraisals. Exceptional Children, 62(3), pp.237-248.

van Daal, J., Verhoeven L., y van Balkom, H. (2007). Behaviour problem in children with language impairment. Journal of child psychology and psychiatry, 48, pp.1139-1147.

Vigotsky, L. S. (1986). Aprendizaje y desarrollo intelectual en la edad escolar. En Luria-LeontievVigotsky. Psicología y pedagogía. Madrid: Akal.

Vilaseca, R. (2004). La influencia del lenguaje de los adultos en el desarrollo morfosintáctico de niños pequeños con Síndrome de Down. Anuario de Psicología, 35(1), pp.87-105.

Vilaseca, R.M. y Del Río (1997). La intervención en el área del lenguaje: un modelo interactivo y naturalista. Infancia y Aprendizaje, vol.76, pp.6-18.

Warren, S. F., y Kaiser, A. P. (1986). Incidental language teaching: A critical review. Journal of Speech and Hearing Disorders, 51, pp.291-299.

Weistuch, L., y Brown, B. (1987). Motherese as therapy: A program and its dissemination. Child Language: Teaching and Therapy, 3, pp.58-71.

Weistuch, L., y Lewis, M. (1985). The language interaction intervention project. Analysis and Intervention in Developmental Disabilities, 5, pp.97-106.

Yoder, P., y Warren, S. (2002). Effects of prelinguistic Milieu Teaching and parent responsivity education on dyads involving children with intellectual disabilities. Journal of Speech, Language, and Hearing Research, 45, pp.1158-1174.

Zaidman-Zait, A., y Young, R. (2008). A parental involvement in the habilitation process following children's cochlear implantation: an action theory. Journal of deaf studies and deaf education, vol.13 (2), pp.193-214. 
ANEXO 1. Estudios incluidos en la revisión.

Estudios que miden la eficacia de los programas centrados en la familia

\begin{tabular}{|c|c|c|c|}
\hline Estudio & Población & $\begin{aligned} \text { Tipo } & \text { de } \\
\text { tratamiento } & \end{aligned}$ & Resultados \\
\hline Bowen y Cupples, 2004 & Niños con TEL & $\begin{array}{l}\text { Educación familiar, } \\
\text { tareas metaanalíticas, } \\
\text { entrenamiento fonético, } \\
\text { trabajo en el hogar. }\end{array}$ & $\begin{array}{l}\text { Mejoras en aspectos } \\
\text { fonéticos }\end{array}$ \\
\hline Mallard, 1998 & Niños con TEL & $\begin{array}{l}\text { Entrenamiento en reso- } \\
\text { lución de problemas y } \\
\text { habilidades sociales }\end{array}$ & $\begin{array}{l}\text { Mejora en el habla en el } \\
82 \% \text { de los niños, de- } \\
\text { cremento en la asisten- } \\
\text { cia a terapia logopédica }\end{array}$ \\
\hline $\begin{array}{l}\text { Mathews, Williams y } \\
\text { Pring, } 1997\end{array}$ & Niños con TEL & $\begin{array}{l}\text { Entrenamiento en habi- } \\
\text { lidades de fluencia }\end{array}$ & $\begin{array}{l}\text { Mejoras en aspectos } \\
\text { fonéticos }\end{array}$ \\
\hline $\begin{array}{l}\text { Millard, Nicholas } \quad \mathrm{y} \\
\text { Cook, 2008 }\end{array}$ & Niños con TEL & $\begin{array}{l}\text { Entrenamiento en pau- } \\
\text { tas de interacción }\end{array}$ & $\begin{array}{l}\text { Mejora en el patrón de } \\
\text { tartamudeo }\end{array}$ \\
\hline $\begin{array}{l}\text { Fey, Cleave, Long y } \\
\text { Hughes, } 1993\end{array}$ & Niños con TEL & $\begin{array}{lr}\text { Programa } & \text { Focused } \\
\text { Stimulation; } & \text { Entrena- } \\
\text { miento en estrategias de } \\
\text { interacción }\end{array}$ & $\begin{array}{l}\text { Mejoras en nivel de } \\
\text { estructura silábica e } \\
\text { inventario consonántico }\end{array}$ \\
\hline $\begin{array}{l}\text { Girolametto, Pearce y } \\
\text { Weitzman, 1996; Mo- } \\
\text { ller, Probst y Hess, } \\
2008\end{array}$ & Niños con TEL & $\begin{array}{l}\text { Programa Hanen; En- } \\
\text { trenamiento en estrate- } \\
\text { gias de interacción }\end{array}$ & $\begin{array}{l}\text { Aumento en el vocabu- } \\
\text { lario y la producción y } \\
\text { comprensión lingüística }\end{array}$ \\
\hline $\begin{array}{l}\text { Chao, Bryan, Burstein y } \\
\text { Ergul, } 2006\end{array}$ & Niños con TEL & Programa CBLA & $\begin{array}{l}\text { Mejoras en el lenguaje } \\
\text { expresivo }\end{array}$ \\
\hline Gibbard, 1994 & Niños con TEL & $\begin{array}{l}\text { Parent Group Language } \\
\text { Intervention; Entrena- } \\
\text { miento en habilidades } \\
\text { lingüísticas }\end{array}$ & $\begin{array}{l}\text { Mejoras en lenguaje } \\
\text { expresivo }\end{array}$ \\
\hline $\begin{array}{l}\text { Pearce, Girolametto y } \\
\text { Weitzman, } \\
\text { Lederer, 2001 }\end{array}$ & Niños con TEL & $\begin{array}{l}\text { Focused Stimulation; } \\
\text { Entrenamiento estrate- } \\
\text { gias de interacción }\end{array}$ & $\begin{array}{l}\text { Aumento en el nivel de } \\
\text { vocabulario }\end{array}$ \\
\hline $\begin{array}{l}\text { Buschmann, Jooss, } \\
\text { Rupp, Fedhusen, Pietz } \\
\text { y Philippi, } 2009\end{array}$ & Niños con TEL & $\begin{array}{l}\text { Programa Heidelberg } \\
\text { Parent-based Language } \\
\text { Intervention }\end{array}$ & $\begin{array}{l}\text { Mejoras en habilidades } \\
\text { expresivas }\end{array}$ \\
\hline $\begin{array}{l}\text { Hemmeter y Kaiser, } \\
\text { 1994; Kaiser y Han- } \\
\text { cock, } 2003\end{array}$ & Niños con TEL & $\begin{array}{l}\text { Programa Enhanced } \\
\text { Milieu Teaching; }\end{array}$ & $\begin{array}{l}\text { Aumento en vocabula- } \\
\text { rio receptivo }\end{array}$ \\
\hline $\begin{array}{l}\text { Fey, Cleave, Long y } \\
\text { Hughes, } 1993\end{array}$ & Niños con TEL & $\begin{array}{ll}\text { Programa } & \text { Focused } \\
\text { Stimulation } & \end{array}$ & $\begin{array}{l}\text { Mejora en habilidades } \\
\text { morfosintácticas (medi- } \\
\text { da en DSS) }\end{array}$ \\
\hline $\begin{array}{l}\text { Kaiser, Hancock y } \\
\text { Trent, 2007; Kaiser y } \\
\text { Hester, 1994; Peterson, } \\
\text { Carta y Greenwood, } \\
2005\end{array}$ & Niños con TEL & $\begin{array}{l}\text { Programa Enhanced } \\
\text { Milieu Teaching; Mo- } \\
\text { delaje de conducta } \\
\text { verbal, enseñanza inci- } \\
\text { dental en contextos } \\
\text { naturales }\end{array}$ & $\begin{array}{l}\text { Aumento en la produc- } \\
\text { ción sintáctica }\end{array}$ \\
\hline $\begin{array}{l}\text { Coulter y Gallhager, } \\
2001\end{array}$ & Niños con TEL & Programa Hanen & $\begin{array}{l}\text { Mejora en habilidades } \\
\text { conversacionales }\end{array}$ \\
\hline $\begin{array}{l}\text { McDonald y } \quad \text { Wilke- } \\
\text { ning, } 1994\end{array}$ & Niños con TEL & 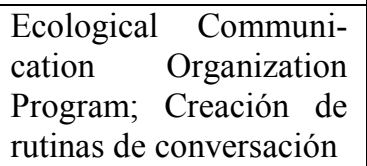 & $\begin{array}{l}\text { Mejora en habilidades } \\
\text { conversacionales }\end{array}$ \\
\hline
\end{tabular}




\begin{tabular}{|c|c|c|c|}
\hline $\begin{array}{l}\text { Mobayed, Collins, } \\
\text { Stranggis, Schuster y } \\
\text { Hemmeter, } 2000\end{array}$ & Niños con TEL & $\begin{array}{l}\text { Responsivity Education } \\
\text { and Prelinguistic Milieu } \\
\text { Teaching; Enseñanza } \\
\text { incidental }\end{array}$ & $\begin{array}{l}\text { Mejoras en habilidades } \\
\text { sociolingüísticas }\end{array}$ \\
\hline $\begin{array}{l}\text { Crowe, Norris y Hoff- } \\
\text { man, } 2000\end{array}$ & Niños con TEL & $\begin{array}{l}\text { Entrenamiento a padres } \\
\text { en habilidad para contar } \\
\text { cuentos }\end{array}$ & $\begin{array}{l}\text { Mejora en toma de } \\
\text { turnos e inicio de actos } \\
\text { comunicativos }\end{array}$ \\
\hline $\begin{array}{lll}\text { Bernhardt, } & \text { Smith } & \text { y } \\
\text { Smith, 1992 } & & \\
\end{array}$ & Niños con TEL & $\begin{array}{l}\text { Entrenamiento en habi- } \\
\text { lidades lingüísticas }\end{array}$ & $\begin{array}{l}\text { Mejora en habilidades } \\
\text { conversacionales }\end{array}$ \\
\hline $\begin{array}{l}\text { Rocha, Schreibonan, y } \\
\text { Stahmer, } \\
\text { Ingersoll, } \\
\text { Dvortcsak, } \\
\text { Schertz y Odom, } 2007 \text {; } \\
2006 ;\end{array}$ & Niños con autismo & $\begin{array}{l}\text { Entrenamiento en habi- } \\
\text { lidades de atención } \\
\text { conjunta }\end{array}$ & $\begin{array}{l}\text { Aumento en inicio y } \\
\text { respuesta de actos de } \\
\text { atención conjunta }\end{array}$ \\
\hline $\begin{array}{l}\text { Mahoney y Perales, } \\
2003\end{array}$ & Niños con autismo & $\begin{array}{l}\text { Entrenamiento en estra- } \\
\text { tegias de interacción }\end{array}$ & $\begin{array}{l}\text { Aumento en las puntua- } \\
\text { ciones de atención, } \\
\text { persistencia, e inicio de } \\
\text { episodios de atención } \\
\text { conjunta }\end{array}$ \\
\hline $\begin{array}{l}\text { Aldred, Green } \quad \text { y } \\
\text { Adamas, } 2001\end{array}$ & Niños con autismo & $\begin{array}{l}\text { Programa Social Com- } \\
\text { munication Interven- } \\
\text { tion; Entrenamiento en } \\
\text { rutinas diarias }\end{array}$ & $\begin{array}{l}\text { Mejoras en actos co- } \\
\text { municativos y aumento } \\
\text { vocabulario expresivo }\end{array}$ \\
\hline $\begin{array}{l}\text { Aldred y cols., 2004; } \\
\text { Girolametto, Sussman y } \\
\text { Weitzman, } 2007 ; \\
\text { McConachie, Randle, } \\
\text { Hammal y Le Couteur, } \\
2005\end{array}$ & Niños con autismo & Programa Hanen & $\begin{array}{l}\text { Aumento en producción } \\
\text { de vocabulario }\end{array}$ \\
\hline $\begin{array}{l}\text { Girolametto, Weitzman } \\
\text { y Clements-Baartman, } \\
1998\end{array}$ & $\begin{array}{l}\text { Niños con Síndrome } \\
\text { Down }\end{array}$ & $\begin{array}{l}\text { Programa } \\
\text { Stimulation; Entrena- } \\
\text { miento en estrategias } \\
\text { facilitadoras del lengua- } \\
\text { je }\end{array}$ & $\begin{array}{l}\text { Aumento en producción } \\
\text { de vocabulario }\end{array}$ \\
\hline $\begin{array}{l}\text { Trent-Strainbook, Kai- } \\
\text { ser y Frey, } 2007\end{array}$ & $\begin{array}{l}\text { Niños con Síndrome } \\
\text { Down }\end{array}$ & $\begin{array}{l}\text { Entrenamiento a her- } \\
\text { manos en estrategias } \\
\text { facilitadoras del lengua- } \\
\text { je }\end{array}$ & $\begin{array}{l}\text { Aumento en habilidades } \\
\text { conversacionales }\end{array}$ \\
\hline $\begin{array}{l}\text { Cheseldine y McCon- } \\
\text { keym, } 1979\end{array}$ & $\begin{array}{l}\text { Niños con retraso cog- } \\
\text { nitivo }\end{array}$ & $\begin{array}{l}\text { Putting Two Words } \\
\text { Together }\end{array}$ & $\begin{array}{l}\text { Aumento de vocabula- } \\
\text { rio, combinación de } \\
\text { palabras y densidad } \\
\text { léxica }\end{array}$ \\
\hline Yoder y Warren, 2002 & $\begin{array}{l}\text { Niños con retraso cog- } \\
\text { nitivo }\end{array}$ & $\begin{array}{l}\text { Responsivity Education } \\
\text { and Prelinguistic Milieu } \\
\text { Teaching }\end{array}$ & $\begin{array}{l}\text { Aumento de vocabula- } \\
\text { rio, combinación de } \\
\text { palabras y densidad } \\
\text { léxica }\end{array}$ \\
\hline $\begin{array}{l}\text { Girolametto, } 1988 ; \\
\text { Tannock, Girolametto y } \\
\text { Siegel, } 1992\end{array}$ & $\begin{array}{l}\text { Niños con retraso cog- } \\
\text { nitivo }\end{array}$ & Programa Hanen & $\begin{array}{l}\text { Incremento en habilida- } \\
\text { des conversacionales, } \\
\text { vocabulario y producti- } \\
\text { vidad lingüística }\end{array}$ \\
\hline $\begin{array}{l}\text { Pennington, Golbart y } \\
\text { Marshall, 2004 }\end{array}$ & $\begin{array}{l}\text { Niños con parálisis } \\
\text { cerebral }\end{array}$ & $\begin{array}{l}\text { Entrenamiento en estra- } \\
\text { tegias de interacción }\end{array}$ & $\begin{array}{l}\text { Aumento en habilidades } \\
\text { comunicativas }\end{array}$ \\
\hline $\begin{array}{l}\text { Zaidman-Zait y Young, } \\
2007\end{array}$ & $\begin{array}{l}\text { Niños con sordera e } \\
\text { hipoacusia }\end{array}$ & $\begin{array}{l}\text { Entrenamiento dirigido } \\
\text { a objetivos }\end{array}$ & $\begin{array}{l}\text { Mejora en el proceso de } \\
\text { habilitación tras im- } \\
\text { plante coclear }\end{array}$ \\
\hline Kargin, 2004 & Niños sordos & Entrenamiento a padres & $\begin{array}{l}\text { Mejora en habilidades } \\
\text { comunicativas }\end{array}$ \\
\hline McConnell, 1974 & Niños sordos & $\begin{array}{l}\text { Bill Wilkerson Hearing } \\
\text { and Speech Center } \\
\text { Program }\end{array}$ & $\begin{array}{l}\text { Mejora en habilidades } \\
\text { comunicativas }\end{array}$ \\
\hline
\end{tabular}




\begin{tabular}{|l|l|l|l|}
\hline García y Turk, 2007 & Niños sordos & $\begin{array}{l}\text { Programa Webster- } \\
\text { Straton modificado }\end{array}$ & $\begin{array}{l}\text { Mejora en habilidades } \\
\text { comunicativas }\end{array}$ \\
\hline Greenburg, 1982 & Niños sordos & $\begin{array}{l}\text { The Counseling and } \\
\text { Home Training Pro- } \\
\text { gram }\end{array}$ & $\begin{array}{l}\text { Mejora en habilidades } \\
\text { comunicativas }\end{array}$ \\
\hline $\begin{array}{l}\text { Harrigan y Nikolopou- } \\
\text { los, 2002 }\end{array}$ & Niños sordos & $\begin{array}{l}\text { Entrenamiento en estra- } \\
\text { tegias de gestión de la } \\
\text { conversación }\end{array}$ & $\begin{array}{l}\text { Mejora en habilidades } \\
\text { conversacionales }\end{array}$ \\
\hline $\begin{array}{l}\text { Girolametto, 1988; } \\
\text { Tannock, Girolametto y } \\
\text { Siegel, 1992 }\end{array}$ & Niños sordos & Programa Hanen & $\begin{array}{l}\text { Mejora habilidades } \\
\text { comunicativas y au- } \\
\text { mento vocabulario }\end{array}$ \\
\hline
\end{tabular}

Estudios que comparan la eficacia de los programas centrados en la familia y la terapia tradicional

\begin{tabular}{|l|l|}
\hline Estudio & Resultados \\
\hline Baxendale y Hesketh, 2003 & $\begin{array}{l}\text { Ganancias significativas con ambos tratamientos, } \\
\text { sin diferencias entre ambos }\end{array}$ \\
\hline Law, Garrett y Nye, 2004 & $\begin{array}{l}\text { Ganancias significativas con ambos tratamientos, } \\
\text { sin diferencias entre ambos }\end{array}$ \\
\hline Fey y cols., 1993 & $\begin{array}{l}\text { Ganancias significativas con ambos tratamientos, } \\
\text { aunque mayor consistencia y mantenimiento de } \\
\text { resultados en terapia tradicional }\end{array}$ \\
\hline Gibbard, Coglan y Mcdonald, 1994;2004 & $\begin{array}{l}\text { Ganancias significativas con ambos tratamientos, } \\
\text { aunque mayor rendimiento en grupos tratados en } \\
\text { familia }\end{array}$ \\
\hline
\end{tabular}

